\title{
Computerised paediatric asthma quality of life questionnaires in routine care
}

\author{
H Mussaffi*, R Omer*, D Prais, M Mei-Zahav, T Weiss-Kasirer, Z Botzer, H Blau
}

Arch Dis Child 2007;92:678-682. doi: 10.1136/adc.2006.111971

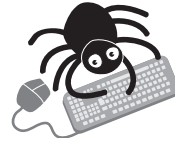

Supplemental files 1 and 2 are available at http:// adc.bmi.com/ supplemental

See end of article for authors' affiliations

Correspondence to: Dr Hannah Blau, Pulmonary Unit, Schneider Children's Medical Center of Israel, 14 Kaplan St, Petah Tikva 49202, Israel;

hblau@post.tau.ac.il

Accepted 2 April 2007

Published Online First

11 April 2007
Background: Asthma quality of life questionnaires are not readily incorporated into clinical care. We therefore computerised the Paediatric Asthma Quality of Life Questionnaire (standardised) (PAQLQ(S)) and the Paediatric Asthma Caregivers Quality of Life Questionnaire (PACQLQ), with a colour-coded printed graphical report.

Objectives: To (a) assess the feasibility of the electronic questionnaires in clinical care and (b) compare the child's PAQLQ scores with the parent's score, physician's clinical score and spirometry.

Methods: Children with asthma were given a clinical severity score of 1-4 (increasing severity) and then completed the PAQLQ(S) electronically (scores 1-7 for increasing quality of life in emotional, symptoms and activity limitation domains) followed by spirometry and physician review. Parents completed the PACQLQ. Inclusion criteria required fluent Hebrew and reliable performance of spirometry. Children with additional chronic diseases were excluded.

Results: 147 children with asthma aged 7-17 years completed PAQLQs and 115 accompanying parents completed PACQLQs, taking $8.3(4.3-15)$ and $4.4(1.5-12.7)$ min, respectively (mean (range)). Graphical reports enabled physicians to address quality of life during even brief visits. Children's (PAQLQ) and parents' (PACQLQ) total scores correlated $(r=0.61, p<0.001)$, although the children's median emotional score of 6.3 was higher than their parents' $5.7(p<0.001)$, whereas median activity limitation score was lower than their parents': 5.0 and 6.8 , respectively $(p<0.001)$. No correlation was found with physician's clinical score or spirometry.

Conclusions: Electronic PAQLQs are easy to use, providing additional insight to spirometry and physician's assessment, in routine asthma care. Future studies must assess impact on asthma management.
A sthma is the most common chronic disease of childhood and adolescence and its impact on the child and their family is far reaching. ${ }^{1}$ In evaluating patients with asthma, we generally rely on reported clinical symptoms, examination and physiological measures such as pulmonary function tests, although these do not directly assess the effect of asthma on daily living. Quality of life is increasingly recognised as an important health measure, especially in chronic diseases such as asthma. ${ }^{23}$

The Paediatric Asthma Quality of Life Questionnaire (PAQLQ) for children, ${ }^{4}$ its standardised version $(\operatorname{PAQLQ}(S))^{5}$ and the Paediatric Asthma Caregivers Quality of Life Questionnaire (PACQLQ) ${ }^{6}$ for their parents or caregivers have been well validated and are perhaps the most widely recognised measures for paediatric asthma quality of life to date. However, quality of life questionnaires, including the PAQLQ, have been used mainly in clinical research ${ }^{3}$ and few publications describe their impact in the "real world" clinical setting of routine asthma clinics. ${ }^{7}$ This may reflect the difficulty of incorporating the information they provide into busy asthma clinics.

As computers and computerised databases become more and more common, the advantages of an electronic or computerised questionnaire are clear. Results can be automatically transformed to graphical formats for instant visual interpretation by the clinician and longitudinal trend follow-up, as well as correlation with other measures such as lung function tests. In addition, they can be automatically incorporated into computerised charts and clinic databases which are rapidly replacing paper patient records worldwide. Electronic versions have been found to be as valid as paper versions in both adults and children and more popular with patients. ${ }^{8} 9$ To our knowledge, there are no published data using electronic questionnaires for children in routine clinical practice.
We recently developed electronic Hebrew versions of the PAQLQ and PACQLQ, which can be used on standard personal computers. Although the interface to the patient and printed report would require language-specific adaptation from the paper versions, the electronic aspects of our measure are universally applicable.

In this study we sought to assess the feasibility of these questionnaires for routine paediatric asthma care. We compared the PAQLQ scores of children with that of their parents as well as with a physician's clinical asthma score and pulmonary function tests.

\section{METHODS}

\section{Subjects}

Children and adolescents diagnosed with asthma and attending the Pulmonary Unit at Schneider Children's Medical Center of Israel together with their parents or caregivers, were invited to complete the PAQLQ during routine visits. Our subjects were aged 7-17 years, the age for which these questionnaires are designed. We included only paired questionnaires completed by both the child and the caregiver in this study. Families were from both urban and rural communities and of varied socioeconomic backgrounds. Inclusion criteria required a diagnosis of asthma according to the National Heart Lung and Blood Institute Guidelines for the Diagnosis and Management of Asthma, ${ }^{10}$ fluent command of Hebrew and the ability to reliably perform spirometry. Children with other chronic diseases in addition to asthma were excluded.

Abbreviations: PACQLQ, Paediatric Asthma Caregivers Quality of Life Questionnaire; PAQLQ(S), Paediatric Asthma Quality of Life Questionnaire (standardised) 

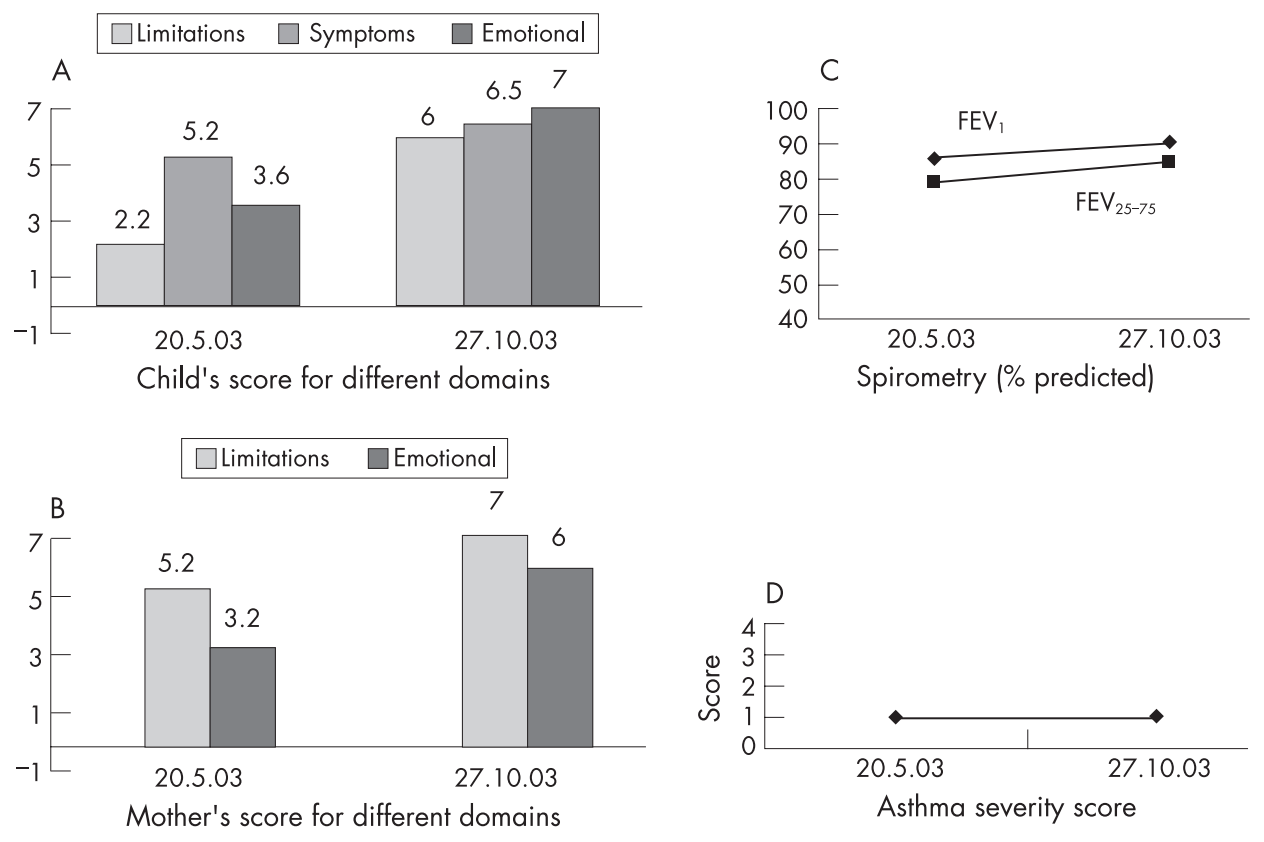

Figure 1 An example of a printed report including graphical display of $(A)$ the electronic PAQLQ(S) for a 9-year-old boy with asthma and (B) the electronic PACQLQ for his mother (score 1-7 for improving quality of life). The report also includes (C) results of pulmonary function tests (FEV $)_{1}$ and (D) the physician's clinical severity score (1-4 for increasing severity). The report is produced immediately on completion of tests and is available for physician review in the clinic.

\section{The electronic version of the PAQLQ}

The PAQLQ(S) and the PACQLQ have been translated into many languages including Hebrew, with stringent validation. ${ }^{11}$ The PAQLQ is scored on a scale of 1 (severely affected) to 7 (unaffected) for each of 23 items covering three domains: activity limitation, symptoms score and the child's emotional reaction to asthma. The activity limitation domain comprises five items relating to play, sports and other daily activities. There were 10 items in the symptoms domain including cough, wheezing and waking at night. The emotional function domain contained eight items, such as being frightened, frustrated or feeling different, being irritable or worried, etc. All items have seven response options. Items are equally weighted and results are expressed as mean score per item for the whole questionnaire, giving a final score in the range of 1-7 for each domain and for the whole instrument, where higher scores indicate better quality of life. The standardised PAQLQ(S) has four "standardised" activities replacing the patient-specific activities in the PAQLQ. ${ }^{4}$ The PACQLQ is scored similarly but includes only two domains: the caregiver's impression of the child's activity limitation and the impact of the child's asthma on the caregiver's emotions. The activity limitation domain contains four items, such as needing to change plans and having sleepless nights because of the child's asthma. The emotional function domain includes nine items, such as feeling helpless or frightened and frustrated and impatient because of the child's asthma. All the questionnaires relate to the situation over the week prior to the clinic visit. There are three forms of the PAQLQ: a self-administered PAQLQ(S) for children and adolescents up to 17 years of age who are able to understand and complete the questionnaires independently, an interviewer-administered PAQLQ(S) for younger children and the PACQLQ to be completed by parents or caregivers accompanying the children.

We undertook to transform the Hebrew versions of both PAQLQ(S)s and the PACQLQ to an electronic medium, strictly adhering to the format of the paper versions. This was reviewed and approved by Mapi Research Institute (Lyon, France) in conjunction with the developer of the original instrument. ${ }^{4-6}$ In the electronic version, the child and parent completed the questionnaires directly on a computer. The interviewer-administered PAQLQ(S) was designed to be administered by an interviewer showing the child coloured response cards corresponding to the questions on the computer screen.

A printed report was formatted to include a colour-coded graphical display of results for each domain of the child's questionnaire as well as total score and a second graph for results of the parent's questionnaire (fig 1). The report also included spirometry results and physician's clinical score.

Technicians performing the pulmonary function tests in our unit's laboratory were trained in the correct methodology of administering the PAQLQ by a clinical psychologist experienced in the administration of quality of life questionnaires. They explained the instructions regarding the PAQLQ to children and their caregivers while the former were awaiting routine pulmonary function tests. The room with the computerised PAQLQ was adjacent to the pulmonary function laboratory so no additional waiting or inconvenience was involved. Parents and children completed questionnaires independently and time taken was recorded.

Results were automatically recorded within an Excel file, together with the physician's clinical score and spirometry results, enabling ready recovery and statistical analysis, both cross-sectional for the group as a whole and longitudinally for any particular subject over time.

\section{Clinical asthma score}

We defined a "real world" clinical asthma score using guideline recommendations. This reflected the accepted routine assessment within the asthma clinic based on reported frequency of attacks, treatment required (both controller and rescue medications), school absence and interference with daily activities as well as examination. Using this information and the Global Initiative for Asthma (GINA) guidelines severity score based on severity of asthma before onset of therapy, physicians scored patients on four levels of severity: (1) mild intermittent asthma, (2) mild persistent asthma, (3) moderate asthma and (4) severe asthma. Scores of 2, 3 and 4 correspond 
to updated definitions of "controlled", "partially controlled" and "uncontrolled" persistent asthma, based on the latest GINA guidelines of 2006. ${ }^{10}$ The physician provided the clinical score before the patients completed the PAQLQ and thus did not know the latter score when making this assessment.

\section{Pulmonary function tests}

Spirometry was performed in the pulmonary function laboratory of the Pulmonary Unit, Schneider Children's Medical Center of Israel, using a Medgraphics spirometer (Medical Graphics, St Paul, MN, USA) and results were expressed as percent of predicted values.

The study was approved by the hospital medical ethics review board and parents and children gave informed consent for participation in the study.

\section{Statistical analysis}

Data were analysed using BMDP statistical software (1993) (Statistical Solutions, Saugus, MA, USA). We used the nonparametric Spearman's rank correlation coefficient (r) to compare the children's emotional and activity limitation scores with that of the parents. This test was also used to compare (a) the physician's clinical severity score with each of the children's individual PAQLQ domains and total quality of life scores, (b) the physician's clinical severity score with each of the parents' PACQLQ domains and total quality of life score and (c) $\mathrm{FEV}_{1 \%}$ and $\mathrm{FEF}_{25-75 \%}$ respectively with each of the children's and parents' individual PAQLQ domains and total quality of life scores. These tests were done for the entire group, as well as for children aged $\leqslant 9$ years old and $>9$ years old as separate groups.

The Wilcoxon matched pairs signed rank test was used to compare the difference between children's and parents' scores. We used Kruskal-Wallis one way analysis of variance to compare total PAQLQ score by the different asthma clinical severity scores.

\section{RESULTS}

\section{Patient recruitment}

A total of 147 children completed the PAQLQ questionnaire over 12 months. Of these, 115 were paired with their accompanying parents who completed the PACQLQ questionnaire. A total of 32 children had no paired data because they came to clinic by themselves or with a family member other than their parent, or because the parents did not speak Hebrew fluently. Successive patients attending the clinic with a diagnosis of asthma who matched the inclusion criteria were recruited. Demographic features, clinical asthma scores and spirometry results are shown in table 1 .

Completion of the PAQLQ for the first time took children $8.3 \pm 2.9 \mathrm{~min}($ mean $\pm \mathrm{SD})$, range $4.3-15 \mathrm{~min}$, and their parents $4.4 \pm 1.9 \mathrm{~min}$ (mean $\pm \mathrm{SD}$ ), range 1.5-12.7 min. Both children and parents easily accepted the electronic system, and there were no difficulties using the computer. Completing the

Table 1 Characteristics of the 115 children in the study

\begin{tabular}{ll}
\hline Male/female (n) & $69 / 46$ \\
Age in years, mean (range) & $12(7-17)$ \\
Children aged 7-9 years, n (\%) & $29(25 \%)$ \\
Children aged 10-17 years, $\mathrm{n}(\%)$ & $86(75 \%)$ \\
Mild intermittent asthma, n (\%) & $21(18 \%)$ \\
Mild persistent asthma, n (\%) & $44(38 \%)$ \\
Moderate persistent asthma, n (\%) & $40(34 \%)$ \\
Severe persistent asthma, n (\%) & $10(9 \%)$ \\
$\mathrm{FEV}_{1} \%$ predicted, mean \pm SD & $86 \pm 14.9$ \\
$\mathrm{FEF}_{25-75 \% \% \text { predicted, mean } \pm \mathrm{SD} .}$ & $78.7 \pm 25.3$ \\
\hline
\end{tabular}

questionnaire did not significantly increase clinic visit time as patients and parents did so while awaiting lung function tests. Waiting time is usually about $15 \mathrm{~min}$.

We found the printed report, available immediately during clinic visits, of great added value for other assessments. An example of a printed PAQLQ report is shown in fig l (see http:// www.archdischild.com/supplemental for supplemental file 1 which gives the results on which this report is based). A 9-yearold boy had a clinical score of 1 representing mild, intermittent asthma, and normal spirometry with little concavity of the flow-volume curve. However, the PAQLQ showed a severe impact of asthma on both the activity limitation and emotional domains. His mother's PACQLQ limitation score was higher (less perception of impairment) but there was a significant maternal emotional impact (score of 3.2 out of 7 for this domain). The child revealed frustration at being unable to qualify for the leading football team, due to exercise-induced asthma during long-distance runs. Pre-training bronchodilator and low-dose inhaled corticosteroids during periods of increased symptoms were recommended. Mother and child were counselled on the uncertainties of living with asthma, the mild nature of the boy's illness and improved coping mechanisms. Longitudinal follow-up showed marked improvement.

We analysed cross-sectional data from families completing the PAQLQ for the first time. Correlations between child and parent for individual and total PAQLQ scores are shown in table 2 . There was significant correlation in all fields including total score $(\mathrm{r}=0.61, \mathrm{p}<0.001)$ and this significance was similar for children $\leqslant 9$ years old and $>9$ years old when analysed separately. Wilcoxon matched pairs analysis showed that the parents' mean emotional score was significantly lower than that of the children $(p<0.001)$, whereas the children's mean activity limitation score was significantly lower than that of the parents $(p<0.001)$. Total PAQLQ scores were compared because they express the overall effect of the asthma on quality of life scores, although they reflect only two domains in caregivers and three domains in the children.

No correlation was found between any of the PAQLQ domains or total scores (for either parents or children) and $\mathrm{FEV}_{1} \%$ (fig 2A) or $\mathrm{FEF}_{25-75 \%}$ (not shown). Similarly, we did not find a correlation between physician's asthma severity score and total PAQLQ score, nor a difference between the four clinical asthma severity groups and total PAQLQ score $(\mathrm{p}=0.086) \quad($ fig $2 \mathrm{~B}) \quad$ (see supplemental file 2 at http:// www.archdischild.com/supplemental for detailed scores).

Table 2 Correlation between the child and parent's emotional, activity limitation and total quality of life (PAQLQ) scores (maximal score: 7) $(n=115)$

\begin{tabular}{lllll}
\hline & $\begin{array}{l}\text { Child's } \\
\text { score, } \\
\text { median } \\
\text { (range) }\end{array}$ & $\begin{array}{l}\text { Parent's } \\
\text { score, } \\
\text { median } \\
\text { (range) }\end{array}$ & $\begin{array}{l}\text { Correlation } \\
\text { coefficient, } \\
(\mathbf{r})^{*}\end{array}$ & $\begin{array}{l}\text { Wilcoxon } \\
\text { signed rank } \\
\text { testt }\end{array}$ \\
\hline $\begin{array}{l}\text { Emotional } \\
\text { score }\end{array}$ & $6.3(2.4-7)$ & $5.7(1-7)$ & $\begin{array}{l}\mathrm{r}=0.53, \\
\mathrm{p}<0.001\end{array}$ & $\mathrm{p}<0.001$ \\
$\begin{array}{l}\text { Activity } \\
\text { limitation }\end{array}$ & $5.0(1.8-7)$ & $6.8(1-7)$ & $\begin{array}{l}\mathrm{r}=0.46, \\
\mathrm{p}<0.001\end{array}$ & $\mathrm{p}<0.001$ \\
$\begin{array}{l}\text { score } \\
\text { Total }\end{array}$ & $5.6(1.9-7)$ & $6.1(1-7)$ & $\begin{array}{l}\mathrm{r}=0.61, \\
\mathrm{p}<0.001\end{array}$ & $\mathrm{p}=0.043$ \\
\hline score & & & $\mathrm{p}<0.001$ \\
\hline
\end{tabular}

*Correlation for the group of children compared to their parents, using Spearman's rank correlation coefficient ( $r$ ).

†The Wilcoxon matched pairs signed rank test comparing the median difference between pairs (child versus parent) for each PAQLQ domain as well as the total score. 

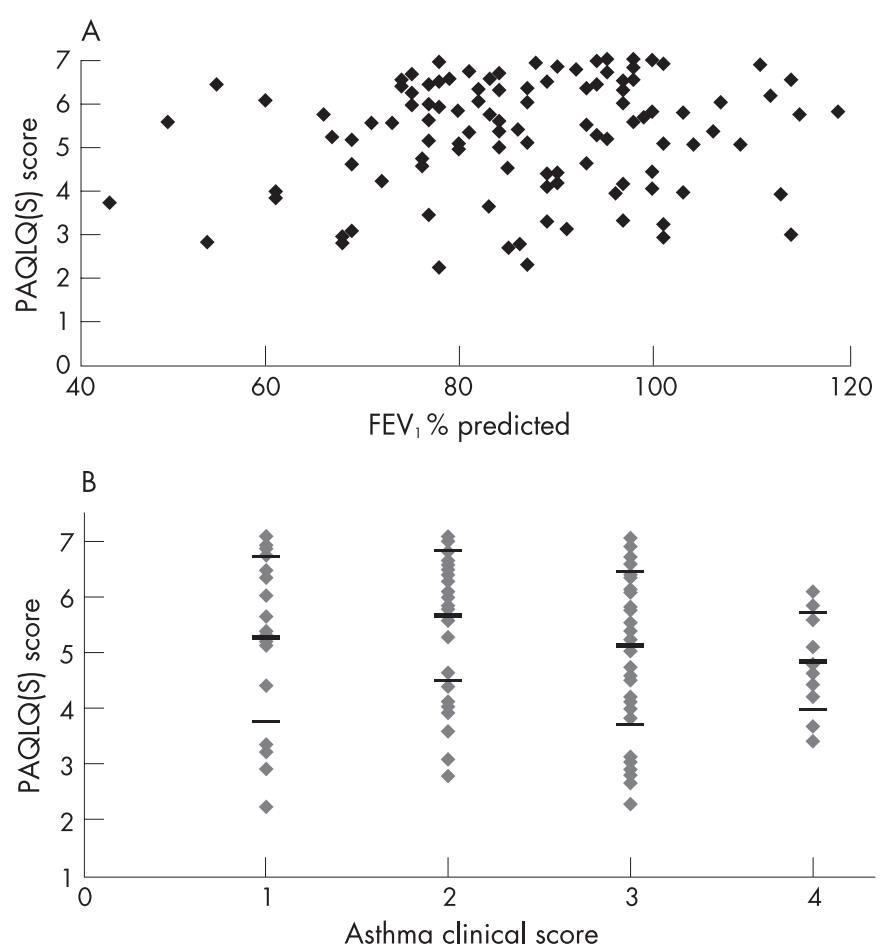

Figure 2 (A) Comparison of the PAQLQ(S) final score for the whole instrument (includes symptoms, emotional and limitations domains) and $\mathrm{FEV}_{1}$ for all 115 children with asthma. Each child is represented by a filled diamond. $r=0.117$ (not significant). (B) Comparison of the PAQLQ(S) final score for the whole instrument (includes symptoms, emotional and limitation domains) for all 115 children with asthma severity score given by the physician. Each child is represented by a shaded diamond. Mean (thick black horizontal line)+standard deviation (thin black horizontal line) are shown for each clinical severity level. $r=-0.17$ (not significant).

\section{DISCUSSION}

Addressing quality of life issues is a critical aspect in managing chronic disease. Indeed, asthma guidelines recommend periodic assessment of the impact of asthma on quality of life. ${ }^{10}$ Several studies highlight the important emotional impact of asthma on adolescents, ${ }^{12}$ children and their families. ${ }^{13}$ Nevertheless, paper versions of quality of life questionnaires have not been incorporated in the routine asthma clinic. The electronic adaptation of quality of life questionnaires has recently been described $^{89}$ and this medium has clear advantages making routine use feasible: it is attractive to children, easy to fill in and practical for routine paediatric asthma care. The time taken was similar to that previously reported for both written and electronic versions. ${ }^{89}$ Unlike the interviewer-administered version for younger children, the self-administered PAQLQ(S) was easier to apply as it did not require an accompanying staff member. Therefore, this would be more feasible with the staff constraints of a busy asthma clinic.

Unlike paper versions, our instrument produced reports, immediately available in a visually striking format and readily incorporated into even a brief clinical visit, and enhancing appreciation of the effect of asthma on the patient's and family's daily life. Importantly, the information provided was not reflected in either spirometry results or the standard physician's assessment. This emphasises the importance of this tool (PAQLQ) in easing the burden of asthma and uncovering obstacles to effective therapy.

We found significant correlations between the parents' and children's scores. However, parents underestimated activity limitation and were more emotionally affected by their child's asthma, as described by others. ${ }^{714}$ Child and parental perception of children's asthma may correlate moderately in younger children ${ }^{16}{ }^{17}$ but poorly in older children. ${ }^{716}{ }^{15}$ However, on dividing our group, we found no difference between those aged 7-9 years and older children. The close correlation in our tertiary referral centre may reflect that fact that $43 \%$ of children had moderate to severe asthma and might be more closely followed by their parents.

We found no correlation between PAQLQ scores and the physicians' asthma severity scoring. Similarly, Williams et al showed little correlation between the beliefs of the healthcare professionals about asthma control, the asthma-related problems of the child, and parental experience of the impact of asthma. ${ }^{7}$ In contrast, good correlation was found during the initial validation of the PAQLQ, but asthma clinical scores incorporated home diaries as well as physician's clinic assessment. ${ }^{46}$ Good correlation was also described in a study of Swedish children aged 7-9 years ${ }^{16}{ }^{17}$ and in older children by Guyatt et al. ${ }^{14}$ Differences may be related to the different design of doctor-assessment scoring. Alternatively, lack of correlation in our study may be due to the small sample size with an under-representation of mild intermittent and severe persistent asthma. Previous stringent validation of the written Hebrew PAQLQ by Mapi Research Institute makes unresponsiveness of this version an unlikely explanation. The physician is frequently unaware of the impact of a child's chronic disease upon the parent despite repeated clinic visits. ${ }^{18}$ Routine use of the electronic PAQLQ may improve the functioning of the parent/ child/physician team in managing asthma by addressing these discrepancies and enabling children and parents to play a greater role in management decisions. The electronic PAQLQs may bring patient concerns to attention and aid the physician in improving the child's asthma control, for example by decreasing limitations of activity. Similarly, the parents with low emotional scores can receive the necessary counselling once clinicians are aware of the situation. Addressing these important areas may improve the ability of children and families to cope with asthma and thus enhance adherence to asthma therapy. ${ }^{19}$

We did not find a correlation between either the children's or parent's scores and the child's $\mathrm{FEV}_{1}$ or $\mathrm{FEF}_{25-75 \%} \%$ predicted (fig 2). This is not surprising as lung function tests during the hospital visit reflect the situation at one instant, while asthma is a disease characterised by much variability. Similarly, Juniper et al found no correlation between PAQLQ or PACQLQ scores and $\mathrm{FEV}_{1} \cdot{ }^{4-6}$ In contrast, there was significant correlation with home monitoring of peak expiratory flow. ${ }^{4}{ }^{16}{ }^{17}$

Our study has several limitations. The electronic version of the PAQLQ has not been validated. Nevertheless, previous studies have shown that electronic questionnaires do not alter responses compared to paper questionnaires, and decrease the number of spoiled responses, as patients cannot accidentally skip questions and must complete each successive step in order. Successful implementation depends on proper instruction in handling the electronic instrument. ${ }^{8}$. We used a clinical asthma score based on guideline recommendations to reflect a physician's assessment during regular asthma care. Although all three senior pulmonologists use this system routinely, no measure of inter-rater reliability was included. In addition, we could not exclude an effect on filling out the PAQLQ of prior clinical assessment by the physician. Finally, socioeconomic influences were not considered in this study. While a previous study showed that these did not affect PAQLQ scores, ${ }^{17}$ another study showed that household income was related to quality of life. ${ }^{20}$ Patients in our hospital clinic are from extremely variable backgrounds. Taking only those subjects where both parents and children completed the questionnaires may have biased our 


\section{What is already known on this topic}

- We generally rely on physician's clinical assessment and on lung function tests for routine paediatric asthma follow-up.

- These measures do not directly assess the daily impact of asthma on the child's and their family's quality of life.

- Paper asthma quality of life questionnaires are useful research tools but clinical use is rarely reported as they are difficult to incorporate into routine care.

\section{What this study adds}

- We describe the development of a computerised Paediatric Asthma Quality of Life Questionnaire (PAQLQ(S)) and Caregivers' PACQLQ, with an immediately available colour-coded printed graphical report.

- Use of this instrument was found to be attractive and practical, making it feasible for routine care in the busy asthma clinic.

- The computerised PAQLQs provided additional insight into the impact of asthma on daily life, which is not reflected in physicians' clinical score or spirometry.

group by not including those subjects where parents did not speak Hebrew, and where adolescents attended clinic alone.

In conclusion, PAQLQ electronic questionnaires are feasible and easy to use, particularly when self-administered, in routine asthma care, and provide additional insight into the effect of asthma on the lives of children and their families. In the future we aim to evaluate their impact on asthma therapy decisions and interventional counselling within the clinic setting, as well as the longitudinal effects on asthma control.

\section{ACKNOWLEDGEMENTS}

We would like to acknowledge the patience and expertise of Ziv Botzer who created the electronic versions of the PAQLQ instruments. We greatly appreciate the support and encouragement of Professor Elizabeth Juniper, the creator of the original PAQLQ instruments. We would like to thank Anne Miege from Mapi Research Institute (Lyon, France) for review and assistance in adapting the Hebrew versions of the three PAQLQ instruments to electronic format.

\section{Authors' affiliations}

H Mussaffi, D Prais, M Mei-Zahav, T Weiss-Kasirer, Z Botzer, H Blau,

Kathy and Lee Graub Cystic Fibrosis Center and Pulmonary Unit, Schneider Children's Medical Center of Israel, Petah Tikva, Israel

R Omer, Department of Psychological Medicine, Schneider Children's

Medical Center of Israel, Petah Tikva, Israel

Competing interests: None.

*HM and RO contributed equally to this study.

Please contact Professor Juniper (juniper@qoltech.co.uk) for permission to access either the original written versions or electronic versions of the questionnaires described in this paper.

\section{REFERENCES}

1 Taylor WR, Newacheck PW. Impact of childhood asthma on health. Pediatrics 1992;90:657-62.

2 Jones PW. Quality of life measurement in asthma. Eur Respir J 1995;8:885-7.

3 Bender BG. Measurement of quality of life in pediatric asthma clinical trials. Ann Allergy Asthma Immunol 1996;77(6):438-45.

4 Juniper EF, Guyatt GH, Feeny DH, et al. Measuring quality of life in children with asthma. Qual Life Res 1996;5:35-46.

5 Juniper EF, Buist AS, Cox FM, et al. Validation of a standardized version of the Asthma Quality of Life Questionnaire. Chest 1999;115:1265-70.

6 Juniper EF, Guyatt GH, Feeny DH, et al. Measuring quality of life in the parents of children with asthma. Qual Life Res 1996:5:27-34.

7 Williams J, Williams K. Asthma-specific quality of life questionnaires in children: are they useful and feasible in routine clinical practice? Pediatr Pulmonol 2003;35:114-18.

8 Bushnell DM, Martin ML, Parasuraman B. Electronic versus paper questionnaires: a further comparison in persons with asthma. J Asthma 2003;40:751-62.

9 Caro JJ Sr, Caro I, Caro J, et al. Does electronic implementation of questionnaires used in asthma alter responses compared to paper implementation? Qual Life Res 2001; 10:683-91.

10 GINA. Global Initiative for Asthma: Guidelines \& Resources, 2006. http:// www.ginasthma.com (accessed 23 April 2007).

11 Juniper E. Measurement of health-related quality of life. Paediatric asthma. http://www.qoltech.co.uk/PaedAsthma.htm (accessed 23 April 2007).

12 Okelo SO, Wu AW, Krishnan JA, et al. Emotional quality of life and outcome in adolescents with asthma. J Pediatr 2004;145:523-9.

13 Rydstom I, Dalheim-Englund A-C, Segesten K, et al. Relations governed by uncertainty: part of life of families of a child with asthma. J Pediatr Nurs 2004;19:85-94.

14 Guyatt GH, Juniper EF, Griffith LE, et al. Children and adult perception of childhood asthma. Pediatrics 1997;99:165-8.

15 Mandhane PJ, McGhan S, Wells H, et al. Parent and child perceptions of asthma quality of life differ (abstract). Am J Respir Crit Care Med 2000;161:A621.

16 Reichenberg K, Broberg AG. The Pediatric Asthma Caregiver's Quality of Life Questionnaire (PACQLQ) in Swedish parents. Acta Paediatr Scand $2001 ; 90: 45-50$

17 Reichenberg K, Broberg AG. Quality of life in childhood asthma: use of the Pediatric Asthma Quality of Life Questionnaire in a Swedish sample of children 7 to 9 years old. Acta Paediatr 2000;89:989-95.

18 Janse AJ, Sinnema G, Uiterwaal CSPM, et al. Quality of life in chronic illness: perceptions of parents and paediatricians. Arch Dis Child 2005;90:486-91.

19 Fiese BH, Fredrick S, Wamboldt FS, et al. Family asthma management routines: connections to medical adherence and quality of life. J Pediatr 2005; 146:171-6.

20 Erickson SR, Munzenberger PJ, Plante MJ, et al. Influence of sociodemographics on the health related quality of life of pediatric patients with asthma and their caregivers. J Asthma 2002;39:107-17. 